\title{
Variação Estacional dos Hormônios Tireoideanos e do Cortisol em Ovinos em Ambiente Tropical $^{1}$
}

\author{
Josiane Maria Cardoso Starling ${ }^{2}$, Roberto Gomes da Silva ${ }^{3}$, João Alberto Negrão ${ }^{4}$, Alex Sandro \\ Campos Maia ${ }^{5}$, Andrea Roberto Bueno ${ }^{6}$
}

\begin{abstract}
RESUMO - Foram estudadas as variações nas concentrações de cortisol e dos hormônios da tireóide de ovinos da raça Corriedale criados em ambiente tropical. Vinte e um ovinos ( 14 machos e sete fêmeas) foram mantidos em uma câmara climática à $45^{\circ} \mathrm{C}$ por nove dias, 8 horas por dia. Registraram-se a temperatura retal (TR) e a frequência respiratória (FR) dos animais, a temperatura ambiente (TA) e a pressão parcial de vapor (PV). Os animais foram posteriormente classificados de acordo com suas respostas quanto à TR e à FR, selecionando-se cinco animais mais aclimatados (grupo A) e cinco animais menos aclimatados (grupo NA) às altas temperaturas do ar. Esses dez animais foram transferidos para um cercado, onde foram registradas a TR e FR duas vezes ao dia e coletadas as amostras sanguíneas uma vez por semana, durante um ano. Foram observadas diferenças nas concentrações de cortisol, de tiroxina $\left(\mathrm{T}_{4}\right)$ e de triiodotironina $\left(\mathrm{T}_{3}\right)$, atribuídas às variações na $\mathrm{TA}$ e na $\mathrm{PV}$. Ocorreram maiores concentrações de cortisol e menores de $\mathrm{T}_{4}$ e de $\mathrm{T}_{3}$ nos períodos de maior TA e/ou PV, sendo que as estimativas dos coeficientes de correlação (r) das variáveis TA e PV com o cortisol foram r $=0,224$ e r $=0,395$, respectivamente. As correlações entre os hormônios tireoideanos e a TA foram negativas $\left(r=-0,156, p a r a T_{4}, e r=-0,151, p a r a T_{3}\right)$, evidenciando que um aumento na TA causou redução na taxa metabólica. As variáveis medidas não diferiram entre os grupos de animais classificados. Portanto, a utilização apenas das variáveis fisiológicas TR e FR como parâmetros para a seleção não é suficiente para se avaliar o grau de aclimatação dos animais ao ambiente tropical.
\end{abstract}

Palavras-chave: ambiente tropical, cortisol, hormônios tireoideanos, ovinos

\section{Seasonal Variation of Thyroid Hormones and Cortisol of Sheep in Tropical Environment}

\begin{abstract}
The variation of cortisol and thyroid hormones in Corriedale sheep bred in a tropical environment was evaluated. Fourteen males and seven females were observed in a climatic chamber at $45^{\circ} \mathrm{C}$ for nine days, eight hours per day. Rectal temperature (TR), respiratory rate (FR), air temperature (TA) and air humidity (PV) were recorded. The animals were ranked according to their responses for TR and FR and then the five highest (A group) and five lowest (NA group) ranked animals were selected. These ten animals were moved to a pen where they were observed along one year for TR and FR two times a day, and blood-sampled once a week. The results showed differences for $\mathrm{T}_{3}, \mathrm{~T}_{4}$, and cortisol concentrations, which were attributed to variations in TA and PV. Cortisol concentrations were higher and $\mathrm{T}_{3}$ and $\mathrm{T}_{4}$ ones were lower under increased TA and PV. Correlations of TA and PV with cortisol values were $\mathrm{r}=.224$ and $\mathrm{r}=.395$ respectively. The negative correlations of TA with $\mathrm{T}_{3}(\mathrm{r}=-.151)$ and $\mathrm{T}_{4}(\mathrm{r}=-.156)$ showed that increase in $\mathrm{TA}$ may be followed by decrease in the metabolic rate. No significant difference for all variables investigated was found between the two groups, thus it is not recommended the use of TR and FR as the only criteria to evaluate sheep performance in tropical environments.
\end{abstract}

Key Words: cortisol, sheep, thyroid hormones, tropical environment

\section{Introdução}

Temperatura e umidade do ar elevadas, assim como intensa radiação solar, são elementos meteorológicos estressantes, geralmente associados ao baixo desempenho de rebanhos criados nas regiões tropicais (Collier et al., 1982). Assim, espera-se que animais mais adaptados às condições inerentes ao clima tropical tenham melhor produtividade, por possuírem características fisiológicas, morfológicas e comportamentais mais adequadas a este ambiente (Roman-Ponce et al., 1981).

A intensidade dos efeitos negativos das temperaturas ambientais elevadas depende da eficiência dos

\footnotetext{
${ }^{1}$ Parte da tese de Doutorado da primeira autora, apresentada à FCAV - UNESP, Jaboticabal, SP, financiada pela FAPESP.

2 Profa Dra. da Faculdade de Medicina Veterinária da UNIFRAN, Franca, SP - Cx Postal 113 - Jaboticabal, SP - cep: $14.870-970$.

E.mail: josistarling@hotmail.com

3 Prof. Dr. do Departamento de Zootecnia - FCAV - UNESP, Jaboticabal, SP. E.mail: robertogomes@netsite.com.br

4 Prof. Dr. do Departamento de Ciências Básicas, Laboratório de Fisiologia Animal, FZEA - USP, Pirassununga, SP. E.mail: jnegrao@usp.br

${ }^{5}$ Doutorando em Zootecnia (Produção Animal). Laboratório de Bioclimatologia do Departamento de Zootecnia - FCAV - UNESP, Jaboticabal,

SP. E.mail: alexsan@fcav.unesp.br

6 Profa Dra. da Faculdade de Medicina Veterinária da UniABC, Santo André, SP. E. mail: andrearbr@uniabc.com.br
} 
mecanismos termorreguladores dos animais (UribeVelasquez et al., 1998). As glândulas adrenais e a tireóide desempenham importantes funções nesse mecanismo de adaptação (Ingraham et al., 1979; Webster et al., 1991) e, segundo McDonald (1980), em animais bem adaptados, respondem rapidamente às mudanças ambientais, proporcionando os ajustes fisiológicos necessários.

Alguns autores demonstraram que, sob altas temperaturas, na fase aguda do estresse térmico, ocorrem elevação da concentração sangüínea de cortisol (Ruckebusch et al., 1991; Alnaimy et al., 1992; McFarlane et al., 1995) e redução na concentração dos hormônios tireoideanos, diminuindo a taxa de produção de calor metabólico (Yousef et al., 1967; Sano et al., 1983; Salem et al., 1991; McNabb, 1995). Outros autores sugerem que os animais domésticos podem suportar algumas condições ambientais adversas, mas alterações consideráveis têm sido observadas em diferentes espécies e indivíduos quanto à capacidade de ajuste às variações sazonais (Nazki \& Rattan, 1991). Portanto, estudar as variações na concentração dos hormônios da tireóide e das adrenais é de extrema importância quando se relaciona estresse térmico e metabolismo (Genuth, 1991; Alnaimy et al., 1992; Dickson, 1996).

Neste contexto, realizou-se este trabalho para analisar, em ovinos da raça Corriedale em ambiente tropical, as possíveis relações existentes entre várias medidas fisiológicas utilizadas como indicativo de desconforto térmico. Na primeira fase (1 $\underline{a}$ fase) do experimento, classificaram-se, via análise multivariada, por meio da avaliação da temperatura retal e da frequência respiratória, os animais experimentais em dois grupos: mais aclimatados (grupo A) e menos aclimatados (grupo NA) ao estresse térmico. Na segunda fase (2 2 fase), confirmou-se, por meio da combinação de várias medidas fisiológicas (temperatura retal, freqüência respiratória, concentração plasmática de $\mathrm{T}_{4}, \mathrm{~T}_{3}$ e cortisol), se a classificação anterior foi eficiente.

\section{Material e Métodos}

O experimento foi realizado em duas fases (1 1 a fase na câmara climática e $2^{\mathrm{a}}$ fase em piquete) e foi conduzido nas instalações do Laboratório de Bioclimatologia Animal do Departamento de Zootecnia da Faculdade de Ciências Agrárias e Veterinárias -
UNESP, Campus de Jaboticabal, SP $\left(21^{\circ} 15^{\prime} 22^{\prime \prime}\right.$ de Latitude Sul, 48 $18^{\prime}$ ' $58^{\prime \prime}$ longitude Oeste e $595 \mathrm{~m}$ de altitude). Durante os experimentos, os animais recebiam água $\mathrm{ad}$ libitum, feno de coast-cross picado (Cynodon dactylon L. Pers) e concentrado comercial (misturados na proporção 40:60), de acordo com a categoria e o estádio fisiológico dos animais (NRC, 1985).

Foram utilizados 21 ovinos da raça Corriedale (sete fêmeas vazias e não-lactantes e 14 machos), com aproximadamente um ano de idade e peso médio de $45 \mathrm{~kg}$, previamente everminados e clinicamente sadios. Na primeira fase (15 a 23 de julho de 1996), os animais foram mantidos em uma câmara climática (com $36 \mathrm{~m}^{2}, 3 \mathrm{~m}$ de pé direito, porta de $2 \mathrm{~m}$ de largura e iluminação interior de luz fluorescente), onde seis radiadores de $1200 \mathrm{~W}$ e um aquecedor central, munido de um exaustor (que retira o ar junto ao solo e lança ar quente no interior da câmara) foram utilizados para manter a temperatura a $45^{\circ} \mathrm{C}$. No centro da câmara, foram instaladas quatro baias (cada uma com 2,6 $\mathrm{m}^{2}$, piso ripado a $25 \mathrm{~cm}$ de altura do solo, dois cochos suspensos e um bebedouro com capacidade de $18 \mathrm{~L}$ ) e um psicrômetro (de bulbos seco e úmido), para o monitoramento da temperatura e da umidade do ar.

Os animais permaneceram nessas baias, na câmara climática, das 8 às $16 \mathrm{~h}$, sendo que os 12 primeiros escolhidos aleatoriamente foram mantidos por quatro dias e os demais, por cinco dias. No restante do dia, os ovinos permaneciam em dois piquetes, cada um com aproximadamente $250 \mathrm{~m}^{2}$, com solo sem cobertura vegetal, cochos cobertos para o fornecimento de volumoso, concentrado e suplemento mineral e com área de sombra para abrigo dos animais.

Três vezes ao dia $(9,14$ e 16 h), registravam-se a freqüência respiratória (FR), por meio da contagem dos movimentos respiratórios por minutos $(\mathrm{mr} / \mathrm{min})$ no flanco dos animais, com o auxílio de um cronômetro; a temperatura retal (TR), utilizando-se termômetros clínico digital $\left({ }^{\circ} \mathrm{C}\right)$; e a temperatura ambiente, por meio de termômetros de bulbos seco e úmido. A umidade do ar, expressa como pressão parcial de vapor $(\mathrm{Pp})$, foi calculada a partir dos registros de temperatura de bulbos úmido e seco, segundo a fórmula:

$$
\mathrm{P}_{\mathrm{p}}\left\{\mathrm{t}_{\mathrm{a}}\right\}=\mathrm{P}_{\mathrm{s}}\left\{\mathrm{t}_{\mathrm{u}}\right\}-\gamma\left(\mathrm{t}_{\mathrm{a}}-\mathrm{t}_{\mathrm{u}}\right),
$$

em que $\operatorname{Pp}\left\{\mathrm{t}_{\mathrm{a}}\right\}=$ pressão parcial de vapor à temperatura $\mathrm{t}_{\mathrm{a}}, \mathrm{kPa} ; \mathrm{P}_{\mathrm{s}}\left\{\mathrm{t}_{\mathrm{u}}\right\}=$ pressão de saturação à temperatura $\mathrm{t}_{\mathrm{u}}, \mathrm{kPa}$, em que $\mathrm{P}_{\mathrm{s}}\left\{\mathrm{t}_{\mathrm{u}}\right\}=0,61078 \times 10^{(7,5 \mathrm{x} \text { tu })}$ / (tu+237,5); $\gamma=$ constante psicrométrica para a temperatura $t_{a}$, obtida em tabela de características do ar, 
$\mathrm{kPa} /{ }^{\circ} \mathrm{C} ; \mathrm{t}_{\mathrm{a}}=$ temperatura de bulbo seco $\left({ }^{\circ} \mathrm{C}\right) ; \mathrm{t}_{\mathrm{u}}=$ temperatura de bulbo úmido $\left({ }^{\circ} \mathrm{C}\right)$.

Conforme os dados de freqüência respiratória e de temperatura retal, subdividiram-se os animais em grupos de indivíduos mais tolerantes e indivíduos menos tolerantes ao estresse por calor, utilizando-se a análise de componentes principais (ACP) como uma análise de agrupamentos (Manly, 1994; Johnson, 1998). A análise estatística dos dados foi realizada por meio do pacote computacional SAS (versão 8.02, 2001), licenciado para a Universidade Estadual Paulista (UNESP).

Apesar de as variáveis não serem expressas nas mesmas unidades, suas variâncias apresentaram homogeneidade e, por isso, optou-se por trabalhar com a matriz de covariâncias, cuja estimativa é apresentada a seguir:

$$
\sum=\left[\begin{array}{cc}
\hat{\sigma}_{1}^{2}(F R) & \operatorname{cov}(F R, T R) \\
\operatorname{cov}(T R, F R) & \hat{\sigma}_{2}^{2}(T R)
\end{array}\right]=\left[\begin{array}{cc}
0,18512 & 0,01725 \\
0,01725 & 0,05425
\end{array}\right]
$$

Os autovalores $\left(\lambda_{\mathfrak{i}}\right)$ e correspondentes autovetores $\mathrm{e}_{\mathrm{i}}=\left[\begin{array}{ll}\mathrm{e}_{\mathrm{i} 1} & \mathrm{e}_{\mathrm{i} 2}\end{array}\right]$ possibilitaram o cálculo dos escores dos dois $(\mathrm{i}=1,2)$ componentes principais $\left(\mathrm{CP}_{\mathrm{ij}}=\mathrm{e}_{\mathrm{i} 1}\right.$ $\left.\mathrm{FR}_{1}+\mathrm{e}_{\mathrm{i} 2} \mathrm{TR}_{1}\right)$ para os 21 animais $(\mathrm{j}=1,2, \ldots, 21)$ :

$$
\begin{array}{lll}
\lambda_{1}=0,187358 & \mathrm{e}_{1}{ }^{\prime}=[0,9917 & 0,1215] \\
\lambda_{2}=0,052017 & \mathrm{e}_{2}{ }^{\prime}=[-0,1285 & 0,9917]
\end{array}
$$

Quatro grupos foram criados utilizando-se os dois componentes principais: grupo $1\left(1^{\circ}\right.$ componente $\geq 0$ e $2^{\circ}$ componente $\left.\geq 0\right)$; grupo $2\left(1^{\circ}\right.$ componente $\geq 0$ e $2^{\circ}$ componente $\left.<0\right)$; grupo $3\left(1^{\circ}\right.$ componente $<0$ e $2^{\circ}$ componente $\geq 0)$ e grupo $4\left(1^{\circ}\right.$ componente $<0$ e $2^{\circ}$ componente $<0$ ), de modo que, no grupo 1 , foram considerados os animais menos tolerantes; nos grupos 2 e 3, os intermediários; e, no grupo 4, os mais tolerantes ao estresse por calor.

Na segunda fase, a partir da análise de componentes principais, selecionaram-se dez animais, que constituíram dois grupos: Grupo A - formado pelos cinco indivíduos (quatro machos e uma fêmea) considerados mais tolerantes às altas temperaturas; e Grupo NA - formado pelos cinco indivíduos (três machos e duas fêmeas) menos tolerantes às altas temperaturas. Nessa fase, verificou-se, por meio da combinação de várias medidas fisiológicas (temperatura retal, freqüência respiratória, concentração plasmática de cortisol, $\mathrm{T}_{4}$ e $\mathrm{T}_{3}$ ), se a classificação dos animais nos grupos A e NA foi eficiente, isto é, se diferenças significativas entre as variáveis para os dois grupos podiam ser detectadas.

Os dados desta fase foram coletados do início de setembro de 1996 ao fim de outubro de 1997. Neste período, os animais também permaneceram nos dois piquetes utilizados na $1 \underline{a}$ fase. O psicrômetro era fixado em um pilar da área de sombra. Três vezes ao dia $(9,14$ e $16 \mathrm{~h}$ ), durante todo o ano, foram registradas as variáveis fisiológicas temperatura retal e freqüência respiratória e as variáveis ambientais temperatura e umidade do ar, efetuando-se o cálculo da pressão parcial de vapor. A partir desses três registros realizados por dia, obteve-se uma média por dia para cada variável mensurada.

Os procedimentos experimentais (coleta de sangue, medidas de temperatura retal e de freqüência respiratória) foram conduzidos em uma cabanha com cobertura de telhas de amianto, cercada com tela, de piso ripado com $10,4 \mathrm{~m}^{2}$ de área e suspensa a $50 \mathrm{~cm}$ de altura do solo.

Uma vez por semana, sempre no mesmo horário $(10 \mathrm{~h}), 5,0 \mathrm{~mL}$ de sangue foram coletados de cada animal por punção da veia jugular, com agulhas hipodérmicas 40 x 12, descartáveis. Imediatamente após a coleta, realizava-se o processamento das amostras e a determinação da concentração hormonal. $\mathrm{O}$ soro foi obtido após coagulação e centrifugação a $0,23478 \mathrm{~g}$ por 15 minutos. Após esse procedimento, eram colhidos $0,25 \mathrm{~mL}$ de soro de cada amostra, para análise hormonal pelo método fluorométrico imunoenzimático no equipamento Baxter Stratus II.

As concentrações séricas de $\mathrm{T}_{3}, \mathrm{~T}_{4}$ e de cortisol foram determinadas utilizando-se kits comerciais (Baxter Diagnostics Inc.) específicos para cada hormônio. Os limites inferior e superior da curva padrão de $\mathrm{T}_{4}$ eram de 5 e $250 \mathrm{ng} / \mathrm{mL}$; de $\mathrm{T}_{3}, 25$ e $8 \mathrm{ng} / \mathrm{mL}$; e de cortisol, $350 \mathrm{ng} / \mathrm{mL}$ e $3 \mathrm{ng} / \mathrm{mL}$, respectivamente. Os coeficientes intra e interdosagens eram de 3,6 e 3,2 , de 5,10 e 1,59 e de 5,1 e 6,5 , respectivamente para $\mathrm{T}_{4}, \mathrm{~T}_{3}$ e cortisol.

O modelo estatístico a seguir foi formulado para avaliação das variáveis experimentais: temperatura retal, freqüência respiratória e dosagens hormonais $\left(\mathrm{T}_{4}, \mathrm{~T}_{3}\right.$ e cortisol):

$$
\begin{gathered}
Y_{\mathrm{ijklm}}=\mu+\mathrm{G}_{\mathrm{i}}+\mathrm{S}_{\mathrm{j}}+\mathrm{GS}_{\mathrm{ij}}+\mathrm{A}_{\mathrm{k}}(\mathrm{GS})_{\mathrm{ij}}+\mathrm{E}_{1}+\mathrm{D}_{\mathrm{m}}(\mathrm{E})_{1} \\
+\mathrm{EG}_{\mathrm{li}}+\mathrm{e}_{\mathrm{ijklm}}
\end{gathered}
$$


em que $Y_{i j k l m}=$ média de três medidas efetuadas no k-ésimo animal do i-ésimo grupo, pertencente ao jésimo sexo, na m-ésima data, da l-ésima estação do ano; $\mu=$ média geral; $g_{i}=$ efeito fixo do i-ésimo grupo $(i=1,2) ; s_{j}=$ efeito fixo do $j$-ésimo sexo $(j=1,2) ; G_{i j}$ = interação aleatória entre o i-ésimo grupo com o jésimo sexo; $A_{k}(G S)_{i j}=$ efeito fixo do k-ésimo animal dentro da interação do i-ésimo grupo com o j-ésimo $\operatorname{sexo}(\mathrm{k}=1, \ldots, 10) ; \mathrm{E}_{1}=$ efeito fixo da l-ésima estação do ano $(1=1, \ldots ., 4) ; D_{m}(E)_{1}=$ efeito fixo da m-ésima data de colheita dentro da l-ésima estação do ano; $\mathrm{EG}_{\mathrm{li}}=$ interação aleatória entre a l-ésima estação do ano com o i-ésimo grupo; $\mathrm{e}_{\mathrm{ijklm}}=$ erro aleatório $\sim \mathrm{N}\left(0, \sigma^{2}\right)$.

Foram estimados os coeficientes de correlação simples entre as variáveis ambientais e fisiológicas medidas durante o período de outubro/96 a outubro/ 97. Também foi feita a comparação entre as médias das variáveis ambientais e fisiológicas por estações do ano pelo teste Tukey a 5\% de probabilidade.

\section{Resultados e Discussão}

Constam na Figura 1 e na Tabela 1 os grupos de animais formados com a utilização da ACP.

Os animais que apresentaram a combinação mais baixa de TR e FR foram considerados os mais tolerantes ao estresse pelo calor (grupo A) e são apresentados no $4^{\circ}$ Quadrante (Figura 1). Os animais menos tolerantes ao estresse pelo calor (grupo NA) apresentaram combinações mais altas de TR e FR (Tabela 1) e são apresentados no $1^{\circ}$ Quadrante da Figura 1. O animal identificado como número $27,1^{\circ}$ Quadrante, (sigla $\mathrm{S}$ ) foi a óbito antes do início da segunda fase experimental por infestação parasitológica. Também na Figura 1 ( $2^{\circ}$ e $3^{\circ}$ Quadrantes $)$ e na Tabela 1 estão apresentados os animais considerados intermediários (I) por apresentarem combinações de TR e FR intermediárias aos extremos dos grupos A e NA selecionados para a $2 \underline{a}$ fase experimental.

Na Tabela 2 encontra-se o resumo das análises de variância para os resultados das variáveis temperatura retal, frequência respiratória e dosagens hormonais ( $\mathrm{T}_{4}, \mathrm{~T}_{3}$ e cortisol), para os dez animais selecionados (grupos A e NA) na 1a fase.

Na Tabela 3 são registradas as médias das variáveis climáticas temperatura e umidade do ar por estações do ano.

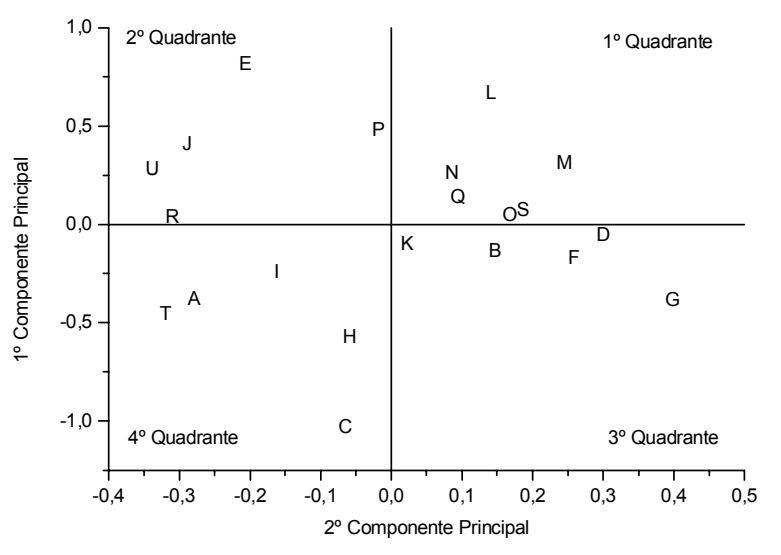

Figura 1 - Dispersão dos 21 animais conforme os valores do primeiro e do segundo componentes principais.

Figure 1 - Scatterplot of the two principal components for the 21 animals.

As médias, estimadas por quadrados mínimos, das variáveis fisiológicas (temperatura retal, frequência respiratória, cortisol, $\mathrm{T}_{4}$ e $\mathrm{T}_{3}$ ) registradas durante a $2^{\mathrm{a}}$ fase são apresentadas na Tabela 4.

$\mathrm{Na}$ Tabela 5 são apresentados os coeficientes de correlação entre as variáveis ambientais e fisiológicas, registradas durante a 2 a fase experimental.

Durante a $2^{\text {a }}$ fase (Tabela 4 ), as médias observadas para temperatura retal mantiveram-se, durante as quatro estações do ano, dentro dos limites considerados fisiológicos para ovinos adultos. Os valores de TR observados são compatíveis com aqueles descritos por alguns pesquisadores (Bianca, 1970; Hahn, 1976; Dukes \& Swenson, 1978; Salem et al., 1982), que, em estudos com ovinos europeus adultos em repouso e à sombra, constataram que a temperatura retal média é de $39,1^{\circ} \mathrm{C}$, podendo ocorrer variações de 38,3 a $40,0^{\circ} \mathrm{C}$. Para a frequência respiratória, Marek \& Mocsy (1963) observaram que, sob condições não estressantes, ovinos lanados apresentam de 12 a 20 movimentos respiratórios por minuto. Entretanto, outros autores (Kolb, 1976; Baccari Jr., 1989) postularam que a amplitude normal seria de 12 a 25 movimentos por minuto, com média de 15 . No entanto, nas duas fases experimentais, a frequência respiratória apresentou médias superiores a 100 movimentos respiratórios por minuto. De maneira geral, ovinos lanados respondem a cargas térmicas excessivas com aumento da fre- 
Tabela 1 - Grupos (A, I, NA) de tolerância ao estresse pelo calor formados com 21 ovinos obtidos a partir da temperatura retal e da freqüência respiratória medidas na câmara climática

Table 1 - Groups (A, I, NA) of heat stress tolerance including 21 sheep obtained considering the rectal temperature and the respiratory frequency, measured in a climatic chamber

\begin{tabular}{|c|c|c|c|c|c|}
\hline $\begin{array}{l}\text { Grupo } \\
\text { Group }\end{array}$ & $\begin{array}{l}\text { Animal } \\
\text { Animal }\end{array}$ & $\begin{array}{l}\text { Sexo } \\
\text { Sex }\end{array}$ & $\begin{array}{c}\text { Sigla } \\
\text { Symbol }\end{array}$ & $\begin{array}{l}\text { TR } \\
T R\end{array}$ & $\begin{array}{l}\text { FR } \\
F R\end{array}$ \\
\hline $\mathrm{A}$ & 1 & $\mathrm{M}$ & A & 40,0250 & 3,46111 \\
\hline A & 3 & M & $\mathrm{C}$ & 40,1533 & 2,79000 \\
\hline A & 12 & $\mathrm{~F}$ & $\mathrm{H}$ & 40,2200 & 3,24222 \\
\hline A & 13 & M & I & 40,1583 & 3,57778 \\
\hline A & 29 & M & $\mathrm{T}$ & 39,9750 & 3,39028 \\
\hline I & 2 & $\mathrm{~F}$ & B & 40,4800 & 3,65556 \\
\hline I & 7 & M & $\mathrm{D}$ & 40,6417 & 3,71528 \\
\hline I & 9 & M & $\mathrm{F}$ & 40,5833 & 3,61250 \\
\hline I & 11 & M & G & 40,6933 & 3,39000 \\
\hline I & 16 & $\mathrm{~F}$ & $\mathrm{~K}$ & 40,3600 & 3,70000 \\
\hline I & 8 & $\mathrm{M}$ & $\mathrm{E}$ & 40,2500 & 4,64306 \\
\hline I & 15 & $\mathrm{M}$ & $\mathrm{J}$ & 40,1167 & 4,24444 \\
\hline I & 23 & M & $\mathrm{P}$ & 40,3917 & 4,26111 \\
\hline I & 26 & $\mathrm{~F}$ & $\mathrm{R}$ & 40,0467 & 3,86111 \\
\hline I & 31 & $\mathrm{M}$ & $\mathrm{U}$ & 40,0467 & 4,10889 \\
\hline NA & 17 & M & $\mathrm{L}$ & 40,5750 & 4,45000 \\
\hline NA & 18 & F & $\mathrm{M}$ & 40,6333 & 4,08111 \\
\hline NA & 19 & M & $\mathrm{N}$ & 40,4667 & 4,04306 \\
\hline NA & 21 & M & $\mathrm{O}$ & 40,5200 & 3,80556 \\
\hline NA & 24 & F & Q & 40,4583 & 3,92222 \\
\hline $\mathrm{NA}^{*}$ & 27 & $\mathrm{M}$ & $\mathrm{S}$ & 40,5417 & 3,84722 \\
\hline
\end{tabular}

$\mathrm{A}=$ Grupo dos animais considerados mais tolerantes ao estresse pelo calor.

$\mathrm{NA}=$ Grupo dos animais considerados menos tolerantes ao estresse pelo calor.

$\mathrm{I}=$ Grupo dos animais intermediários entre A e NA.

*Animal foi a óbito antes da $2^{\mathrm{a}}$ fase experimental.

TR = Temperatura retal

$\mathrm{FR}=$ Freqüência respiratória.

$A=$ Group of animal classified as more adapted to heat stress.

$N A=$ Group of animal classified as less adapted to heat stress.

$I=$ Group of animal classified as intermediary between $A$ and NA.

*Animal died before the second trial.

$T R=$ Rectal temperature.

$F R=$ Frequency rate .

qüência respiratória, de forma a aumentar a dissipação do calor corporal por meio do aquecimento do ar inspirado e da evaporação nas vias respiratórias (Mendes et al., 1976; Baccari Jr., 1989; Silva \& Gaudiosi, 1995), resultando em forte elevação da freqüência respiratória (polipnéia), que pode atingir até 400 movimentos respiratórios por minuto (Mendes et al., 1976). De acordo com Silva (2000), a capa protetora oferecida pelo velo de lã reduz consideravelmente a evaporação por sudação e, portanto, a evaporação respiratória apresenta real importância termorreguladora. Desta forma, parece que os ovinos responderam à elevação da temperatura e da umidade do ar com o aumento da frequência respiratória, de forma a favorecer a manutenção da homeotermia, por meio da evaporação respiratória.

Neste estudo, a frequência respiratória apresentou coeficiente de correlação positivo com a temperatura $\operatorname{retal}(\mathrm{r}=0,52, \mathrm{p}<0,01$ - Tabela 5), indicando ter sido um mecanismo respiratório muito importante para a termólise e a manutenção da homeotermia dos animais.

Ainda na 2 a fase, as fêmeas apresentaram, para a frequência respiratória valores médios superiores aos dos machos (135,6 e 119,3 mr/min.), porém não estatisticamente significativos. O mesmo ocorreu com a temperatura retal, obtendo-se valores de $39,4^{\circ} \mathrm{C}$ para machos e de $39,8^{\circ} \mathrm{C}$ para fêmeas. Não houve efeito de sexo ou grupo para concentrações hormonais mensuradas. Porém, verificou-se efeito significativo das estações do ano sobre os níveis hormonais, evidenciando a influência da ação conjunta das variáveis metereológicas estudadas sobre a fisiologia endócrina e termorreguladora dos animais.

As maiores médias do cortisol foram registradas na primavera e no verão, com valores significativamente maiores $(15,2$ e $14,4 \mathrm{ng} / \mathrm{mL})$ que os encontrados no 
Tabela 2 - Quadrados médios da ANOVA com respectivas significâncias, coeficientes de variação (CV) e de correlação $\left(r^{2}\right)$ das variáveis fisiológicas temperatura retal $\left(T R-{ }^{\circ} \mathrm{C}\right)$, freqüência respiratória (FR - mr./min.) e variáveis hormonais tiroxina $\left(T_{4}-n g / m L\right)$, triiodotironina $\left(T_{3}-n g / m L\right)$ e cortisol $(n g / m L)$

Table 2 - ANOVA means square with respective significance levels, coefficient of variation (CV), and correlation coefficient $\left(r^{2}\right)$ of the physiological variables of rectal temperature $\left(T R-{ }^{\circ} \mathrm{C}\right)$ and respiratory frequency $(F R-r m . / m i n$.$) and the hormonal$ variables thyroxine $\left(T_{4}-n g / m L\right)$, triiodothyronine $\left(T_{3}-n g / m L\right)$ and cortisol $(n g / m L)$

\begin{tabular}{|c|c|c|c|c|c|c|c|c|}
\hline \multirow{3}{*}{$\begin{array}{l}\text { Fonte de variação } \\
\text { Source of variation }\end{array}$} & \multicolumn{2}{|c|}{ TR } & \multicolumn{2}{|c|}{ FR } & \multirow{3}{*}{$\begin{array}{c}\text { Hormônio } \\
\text { Hormone } \\
\text { GL } \\
D F\end{array}$} & \multirow{2}{*}{$\begin{array}{l}\mathrm{T}_{3} \\
\mathrm{QM}\end{array}$} & \multirow{2}{*}{$\begin{array}{l}\mathrm{T}_{4} \\
\mathrm{QM}\end{array}$} & \multirow{3}{*}{$\begin{array}{l}\text { Cortisol } \\
\text { QM } \\
M S\end{array}$} \\
\hline & GL & QM & GL & QM & & & & \\
\hline & $D F$ & $M S$ & $D F$ & $M S$ & & $M S$ & $M S$ & \\
\hline Grupo & 1 & 0,207 & 1 & 2004,293 & 1 & 0,521 & 177,492 & 5,400 \\
\hline \multicolumn{9}{|l|}{ Group } \\
\hline SexoSex & 1 & 15,899 & 1 & 21181,052 & 1 & 0,523 & 210,570 & 4,031 \\
\hline Grupo*sexo & 1 & 2,696 & 1 & 934,312 & 1 & 0,515 & 20,862 & 114,134 \\
\hline Group*sex & & & & & & & & \\
\hline $\begin{array}{l}\text { Animal (grupo*sexo) } \\
\text { Animal (group*sex) }\end{array}$ & 7 & $14,591 *$ & 7 & $26140,381^{*}$ & 6 & $0,992 *$ & $2761,193^{*}$ & $157,855^{*}$ \\
\hline $\begin{array}{l}\text { Estação do ano } \\
\text { Season of the year }\end{array}$ & 3 & $8,329^{*}$ & 3 & $24869,419^{*}$ & 3 & $43,892 * *$ & $7293,411^{*}$ & $351,119 *$ \\
\hline Data (estação do ano) & 225 & $0,503^{*}$ & 224 & $3486,016^{*}$ & 44 & $2,090^{*}$ & $2545,239^{*}$ & $731,474 *$ \\
\hline Date (Season of the year) & & & & & & & & \\
\hline $\begin{array}{l}\text { Grupo*estação do ano } \\
\text { Group*season of the year }\end{array}$ & 3 & $0,415^{*}$ & 3 & $2574,551^{*}$ & 3 & 0,158 & 88,305 & 38,832 \\
\hline ResíduoError & 2048 & 0,0549 & 2039 & 226,52 & 420 & 0,0856 & 57,62 & 24,40 \\
\hline TotalTotal & 2289 & & 2279 & & 479 & & & \\
\hline CV (\%) & & 0,59 & & 12,05 & & 16,70 & 12,98 & 35,52 \\
\hline$r^{2}$ & & 0,76 & & 0,72 & & 0,87 & 0,86 & 0,77 \\
\hline
\end{tabular}

${ }^{*} p<0,01 ;{ }^{* *} p<0,05$.

* $p<0.01 ; * * 0<0.05$.

$\mathrm{GL}$ - graus de liberdade (DF - degrees of freedom).

QM - quadrados médios (mean square).

Tabela 3 - Médias das variáveis climáticas temperatura ambiente (TA - ${ }^{\circ} \mathrm{C}$ ) e pressão parcial de vapor ( $\mathrm{PV}$ - kPa) e variações mínima e máxima por estações do ano

Table 3 - Means of air temperature $\left(T A-{ }^{\circ} \mathrm{C}\right)$ and air humidity $(\mathrm{PV}-\mathrm{kPa})$ and minimum and maximum variations according to the season of the year

\begin{tabular}{lccrrc}
\hline $\begin{array}{l}\text { Estação do ano } \\
\text { Season of the year }\end{array}$ & $\mathrm{N}$ & $\begin{array}{c}\text { Variável } \\
\text { Variable }\end{array}$ & $\begin{array}{c}\text { Média } \\
\text { Mean }\end{array}$ & $\begin{array}{c}\text { Mínimo } \\
\text { Minimum }\end{array}$ & $\begin{array}{c}\text { Máximo } \\
\text { Maximum }\end{array}$ \\
\hline $\begin{array}{l}\text { Primavera } \\
\text { Spring }\end{array}$ & 760 & TA & 28,12 & 19,67 & 35,83 \\
Verão & & PV & 2,43 & 1,58 & 3,54 \\
Summer & 460 & TA & 28,58 & 21,67 & 32,33 \\
Outono & & PV & 2,56 & 2,09 & 2,87 \\
Autumn & \multirow{2}{*}{500} & TA & 24,95 & 18,77 & 30,80 \\
Inverno & & PV & 1,99 & 1,15 & 3,17 \\
Winter & \multirow{2}{*}{570} & TA & 26,85 & 19,73 & 34,60 \\
\hline
\end{tabular}

$\mathrm{N}=$ número de dias avaliados (numbers of days evaluated).

outono e inverno $(\mathrm{p}<0,05)$. Durante essas estações, ocorrem as maiores médias de temperatura do ar e de pressão parcial de vapor (Tabela 3), o que poderia ter influenciado a concentração sanguínea de cortisol, pois a alta umidade do ar, associada à alta temperatura do ar, dificulta o processo de termólise nos animais.

Assim, o aumento nas concentrações médias de cortisol pode ser atribuído à elevação da temperatura ambiente e da pressão parcial de vapor medida nestas estações. Essa argumentação é fortalecida quando se verifica que, durante as estações de temperatura e umidade do ar mais amenas (outono e inverno Tabela 3), foram registrados menores níveis médios de cortisol $(p<0,05)$. Em razão desses fatos, as concentrações de cortisol (Tabela 5) mostraram-se positivamente correlacionados à temperatura ambiente $\mathrm{e}$ 
Tabela 4 - Médias ${ }^{1}$ ajustadas e respectivos erros-padrão para as variáveis temperatura retal $\left(T R\right.$ - $\left.{ }^{\circ} \mathrm{C}\right)$ e frequência respiratória (FR - mr./min.) e para as variáveis hormonais tiroxina $\left(T_{4}-\mathrm{ng} / \mathrm{mL}\right)$, triiodotironina $\left(T_{3}-\mathrm{ng} / \mathrm{mL}\right)$ e cortisol $(\mathrm{ng} / \mathrm{mL})$ por estação do ano, sexo e grupo dos animais

Table 4 - Least square means ${ }^{1}$ and standard errors for the variables rectal temperature $\left(T R-{ }^{\circ} \mathrm{C}\right)$ and respiratory frequency (FR - $\mathrm{mr}$./min.) and the hormonal variables thyroxine $\left(T_{4}-n g / m L\right)$, triiodothyronine $\left(T_{3}-n g / m L\right)$ and cortisol ( $\left.n g / m L\right)$ according to the season of the year, sex and group of the animals

\begin{tabular}{|c|c|c|c|c|c|}
\hline $\begin{array}{l}\text { Efeito } \\
\text { Effect }\end{array}$ & TR & FR & $\mathrm{T}_{4}$ & $\mathrm{~T}_{3}$ & Cortisol \\
\hline $\begin{array}{l}\text { Primavera } \\
\text { Spring }\end{array}$ & $39,75 \pm 0,01^{\mathrm{a}}$ & $131,04 \pm 0,90^{\mathrm{b}}$ & $63,06 \pm 0,65^{\mathrm{a}}$ & $0,97 \pm 0,02^{\mathrm{d}}$ & $15,20 \pm 0,42^{\mathrm{a}}$ \\
\hline $\begin{array}{l}\text { Verão } \\
\text { Summer }\end{array}$ & $39,55 \pm 0,01^{b}$ & $121,08 \pm 1,01^{\mathrm{c}}$ & $61,55 \pm 0,87^{\mathrm{a}}$ & $1,87 \pm 0,03^{\mathrm{c}}$ & $15,42 \pm 0,57^{\mathrm{a}}$ \\
\hline $\begin{array}{l}\text { Outono } \\
\text { Autumn }\end{array}$ & $39,56 \pm 0,01^{b}$ & $122,39 \pm 0,97^{\mathrm{c}}$ & $62,70 \pm 0,69^{a}$ & $2,03 \pm 0,02^{b}$ & $12,34 \pm 0,45^{b}$ \\
\hline $\begin{array}{l}\text { Inverno } \\
\text { Winter } \\
\text { Sexo }\end{array}$ & $39,78 \pm 0,01^{\mathrm{a}}$ & $135,31 \pm 0,96^{\mathrm{a}}$ & $47,04 \pm 0,72^{b}$ & $2,25 \pm 0,03^{\mathrm{a}}$ & $12,29 \pm 0,47^{b}$ \\
\hline $\begin{array}{l}\text { Sex } \\
\text { Fêmeas }\end{array}$ & $39,87 \pm 0,01$ & $135,63 \pm 0,61$ & $59,32 \pm 0,68$ & $1,74 \pm 0,02$ & $13,67 \pm 0,44$ \\
\hline $\begin{array}{l}\text { Females } \\
\text { Machos } \\
\text { Males }\end{array}$ & $39,45 \pm 0,02$ & $119,28 \pm 1,44$ & $57,86 \pm 0,43$ & $1,82 \pm 0,01$ & $13,95 \pm 0,28$ \\
\hline $\begin{array}{l}\text { Grupos } \\
\text { Groups }\end{array}$ & & & & & \\
\hline $\begin{array}{l}\text { A } \\
\text { NA }\end{array}$ & $\begin{array}{l}39,62 \pm 0,01 \\
39,70 \pm 0,02\end{array}$ & $\begin{array}{l}125,10 \pm 0,56 \\
129,81 \pm 1,47\end{array}$ & $\begin{array}{l}57,88 \pm 0,62 \\
59,29 \pm 0,51\end{array}$ & $\begin{array}{l}1,81 \pm 0,02 \\
1,75 \pm 0,02\end{array}$ & $\begin{array}{l}13,85 \pm 0,41 \\
13,78 \pm 0,33\end{array}$ \\
\hline
\end{tabular}

${ }^{1}$ Médias com letras diferentes, na mesma coluna, diferiram $(p<0,05)$ significativamente entre si pelo teste Tukey.

$A=$ Grupo dos animais considerados mais tolerantes ao estresse pelo calor.

$\mathrm{NA}=$ Grupo dos animais considerados menos tolerantes ao estresse pelo calor.

${ }^{1}$ Means followed by the same letter en each column do not differ $(p<0.05)$ by Tukey pairwise comparison method.

$A=$ Group of animal classified as more adapted to heat stress.

$N A=$ Group of animal classified as less adapted to heat stress.

Tabela 5 - Coeficientes de correlação entre as variáveis ambientais e fisiológicas e respectivos níveis de significância para o teste de Ho: correlação $=0$

Table 5 - Correlation coefficients among environmental and physiologic variables and respective significance levels by the Ho: correlation $=0$ test

\begin{tabular}{lcccccc}
\hline & PV & TR & FR & $\mathrm{T}_{4}$ & $\mathrm{~T}_{3}$ & Cortisol \\
\hline TA & $0,41359^{*}$ & $0,23396^{*}$ & $0,36482^{*}$ & $-0,15635^{*}$ & $-0,15101^{*}$ & $0,22368^{*}$ \\
PV & & $0,11428^{*}$ & 0,00527 & $-0,05547$ & $-0,14499^{*}$ & $0,39467^{*}$ \\
TR & & $0,51867^{*}$ & 0,05189 & $-0,06649$ & $0,09145^{* *}$ \\
FR & & & $0,13576^{*}$ & $-0,03312$ & $-0,01735$ \\
$\mathrm{~T}_{4}$ & & & & $-0,17954^{*}$ & $-0,20708^{*}$ \\
$\mathrm{~T}_{3}$ & & & & & $0,24119^{*}$ \\
\hline
\end{tabular}

\section{* $(p<0,01) \quad{ }^{* *}(p<0,05)$}

TA e PV $=$ Temperatura do ar $\left({ }^{\circ} \mathrm{C}\right)$ e pressão parcial de vapor $(\mathrm{kPa})$

$\mathrm{TR}$ e $\mathrm{FR}=\mathrm{Temperatura}$ retal $\left({ }^{\circ} \mathrm{C}\right)$ e frequência respiratória $(\mathrm{mr} . / \mathrm{min}$.)

$\mathrm{T}_{4}=$ Tiroxina $(\mathrm{ng} / \mathrm{mL}) ; \mathrm{T}_{3}=$ Triiodotironina $(\mathrm{ng} / \mathrm{mL})$

${ }^{*}(p<0.01) \quad{ }^{* *}(p<0.05)$

$T A$ and $P V=$ Air temperature $\left({ }^{\circ} \mathrm{C}\right)$ and air humidity $(\mathrm{kPa})$

$T R$ and $F R=$ Rectal temperature $\left({ }^{\circ} \mathrm{C}\right)$ and respiratory frequency ( $\mathrm{mr} . / \mathrm{min}$.)

$T_{4}=$ Thyroxine $(\mathrm{ng} / \mathrm{mL}) ; T_{3}=$ Triiodothyronine $(\mathrm{ng} / \mathrm{mL})$.

à pressão parcial de vapor $(0,223$ e 0,395 , respectivamente $(\mathrm{p}<0,01)$, indicando que possivelmente a elevação da temperatua correspondeu ao aumento do desconforto térmico dos animais e, portanto, à maior secreção de cortisol.
As concentrações de cortisol medidas neste estudo (Tabela 4) foram próximas aos valores médios de 6 a $14 \mathrm{ng} / \mathrm{mL}$ descritos por Encarnação (1989), porém, inferiores ao valor médio de $20 \mathrm{ng} / \mathrm{mL}$ relatado por Hargreaves \& Hutson (1990) e Minton et al. (1995), 
que dosaram esse hormônio em ovinos, sem imposição de nenhum tipo de estresse. Contudo, Hargreaves \& Hutson (1990), ao utilizarem a tosquia como agente estressor, observaram um pico muito importante $(72,7 \mathrm{ng} / \mathrm{mL})$ de cortisol. Esse pico foi semelhante ao nível máximo $(73,0 \mathrm{ng} / \mathrm{mL})$ encontrado nesta pesquisa, mas foi bem superior às médias obtidas por estação do ano (Tabela 4).

No inverno e no outono, como esperado, ocorreram as menores médias de temperatura e de umidade do ar (Tabela 3) e, conseqüentemente, foram observadas (Tabela 4) as maiores concentrações médias de triodotironina $(2,03$ e $2,25 \mathrm{ng} / \mathrm{mL}$, respectivamente, $\mathrm{p}<0,05)$. Observou-se, assim, uma resposta termogênica desse hormônio às temperaturas do ar mais amenas ocorridas durante essas estações. Conseqüentemente, no inverno, com a maior demanda do $\mathrm{T}_{3}$, houve maior conversão do $\mathrm{T}_{4}$ em $\mathrm{T}_{3}$, ocorrendo redução na concentração média do $\mathrm{T}_{4}(47,04 \mathrm{ng} / \mathrm{mL})$.

As concentrações médias de cortisol foram semelhantes para essas duas estações e menores em relação às das estações anteriores (primavera e verão), provavelmente em razão da redução na temperatura e na umidade do ar.

$\mathrm{Na}$ primavera e no verão, foram estimadas as maiores médias de temperatura (Tabela 3) e umidade do ar neste estudo. Nestas estações também foram observadas maiores concentrações médias de cortisol e menores de $\mathrm{T}_{3}$, (Tabela 4), entre as estações estudadas. Esses resultados confirmam as suposições de Yousef et al. (1967), Genuth (1991), Alnaimy et al. (1992), McFarlane et al. (1995) e Dickson (1996) de que ocorre redução na atividade tireoideana em resposta ao incremento da carga térmica sobre os animais, ao mesmo tempo em que se elevam os níveis de cortisol.

Os níveis médios de $\mathrm{T}_{3}$ neste estudo foram superiores à média registrada por Nascimento (1994), de $0,82 \mathrm{ng} / \mathrm{mL}$, ao verificar a ação do estresse calórico sobre o nível de $\mathrm{T}_{3}$ em ovinos Corriedale adultos mantidos em câmara climática à temperatura de $45^{\circ} \mathrm{C}$. Fraser \& McNeilly (1980), ao analisarem semanalmente os níveis plasmáticos de $\mathrm{T}_{3}$ em ovelhas, encontraram variações de 0,7 a $1,9 \mathrm{ng} / \mathrm{mL}$. Neste estudo, o menor valor médio quantificado para $\mathrm{T}_{3}$ foi semelhante $(0,97 \mathrm{ng} / \mathrm{mL})$, porém, a variação foi maior $(0,97$ a 2,25 ng/mL). Segundo Christon (1988), a variação das concentrações de $\mathrm{T}_{3}$ está diretamente relacionada à temperatura ambiente e associada à taxa de produção de calor metabólico, caracterizando uma situação de estresse calórico. Neste trabalho, as variações observadas nos níveis de $\mathrm{T}_{3}$, nas diferentes estações do ano, favoreceram esta argumentação.

As concentrações médias de $\mathrm{T}_{4}$ foram semelhantes aos valores médios descritos por Wallace (1979), em estudo realizado em condições climáticas semelhantes. Quando comparadas a outros trabalhos envolvendo ovinos em diferentes condições ambientais (Sutherland \& Irvine, 1973; Reap et al., 1978; Ruckebusch et al., 1991), as médias de $\mathrm{T}_{4}$ (Tabela 4) observadas nesta pesquisa foram ligeiramente maiores, porém, inferiores ao valor médio $(91,88 \mathrm{ng} / \mathrm{mL})$ descrito por Nascimento (1994).

Verificou-se (Tabela 5) que, os coeficientes de correlação obtidos foram baixos, porém, significativos $(p<0,01)$. A frequência respiratória apresentou coeficiente de correlação positivo com a temperatura retal $(r=0,519)(p<0,01)$, sugerindo que esse deve ser um mecanismo respiratório muito importante para termólise e, conseqüentemente, para a manutenção da homeotermia dos animais nas condições em que foram observados.

De modo geral, as temperaturas ambientes apresentaram correlações significativas e negativas com os hormônios tireoideanos, observando-se que, quanto maior a temperatura ambiente e mais alta a umidade do ar, menores os níveis de $\mathrm{T}_{3}$ e $\mathrm{T}_{4}$; e que as concentrações de $T_{3}$ e de $T_{4}$ correlacionaram-se também negativamente com a umidade do ar.

Os níveis de cortisol mostraram-se positivamente correlacionados à temperatura do $\operatorname{ar}(\mathrm{r}=0,224)$ e à pressão parcial de vapor $(\mathrm{r}=0,395)$, evidenciando uma resposta do organismo à elevação da temperatura e da umidade do ar. Esses resultados indicaram que possivelmente a elevação da temperatura e da umidade atmosférica corresponde a um aumento do desconforto térmico dos animais e, portanto, da secreção de cortisol, pois a associação dessas duas variáveis ambientais é uma fonte importante de estresse calórico. Os resultados (Tabela 5) sugerem as suposições usuais de que ocorre redução na atividade tireoideana em resposta ao incremento da carga térmica sobre os animais, ao mesmo tempo em que se elevam os níveis de cortisol.

A concentração de cortisol correlaciou-se positivamente com a concentração de $\mathrm{T}_{3}(\mathrm{r}=0,241)$ e negativamente com a de $\mathrm{T}_{4}(\mathrm{r}=-0,207)(\mathrm{p}<0,01)$. Aparentemente, o cortisol responde mais rapidamente ao estresse térmico, ao passo que o período de latência 
é maior nas respostas do $\mathrm{T}_{4}$ e do $\mathrm{T}_{3}$. Esses resultados também corroboram os descritos por outros autores (Re et al., 1976; Ross et al., 1985; McNabb, 1995). De acordo com Sterling \& Lazarus (1977), o aumento dos níveis dos hormônios tireoideanos na circulação sanguínea durante o estresse calórico inicial ocorre em razão de sua menor utilização, provocando retardamento na redução da taxa de secreção da glândula tireóide.

\section{Conclusões}

Nas condições em que foi realizado este estudo, os resultados permitiram concluir que as variáveis fisiológicas temperatura retal e frequência respiratória, como únicos parâmetros para identificar animais aclimatados ao clima tropical, não expressam suficientemente as condições de adaptabilidade. Nos estudos de adaptação, deve ser considerado o conjunto das respostas fisiológicas e comportamentais dos animais às condições ambientais para adequada avaliação do grau de aclimatação. As concentrações séricas dos hormônios $\mathrm{T}_{4}, \mathrm{~T}_{3}$ e cortisol são afetadas pela temperatura e umidade do ar, de modo que, durante o estresse térmico, há uma resposta mais rápida do cortisol e maior período de latência na resposta dos hormônios tireoideanos. A utilização destes hormônios como indicadores de estresse térmico em estudos de adaptação em ovinos facilita a compreensão dos mecanismos fisiológicos envolvidos nos processos de termólise e homeotermia.

\section{Literatura Citada}

ALNAIMY, A.; HABEEB, M.; FAYAZ, I. et al. Heat stress. In: PHILLIPS, C.; PIGGINS, D. (Eds.) Farm animals and the environment. Wallingford: CAB International, 1992. p.27-47.

BACCARI JR., F. Mecanismos adaptativos de ovinos lanados nos trópicos. In: SIMPÓSIOPAULISTA DE OVINOCULTURA, 1., 1989, Campinas. Anais... Campinas: 1989. p.9-17.

BIANCA, W. Animal response to meteorological stress as a function of age. Biometeorology, v.4, n.1, p.119-22, 1970.

CHRISTON, R. The effect of tropical ambient temperature on growth and metabolism in pigs. Journal of Animal Science, v.66, n.7, p.3112-3123, 1988.

COLLIER, R.J.; BEEDE, D.K.; THATCHER, W.W. et al. Influence of environment and its modification on dairy animal health and production. Journal of Dairy Science, v.65, p.2213-2227, 1982.

DICKSON, W.M. Endocrinologia, reprodução e lactação. Glândulas endócrinas In: SWENSON, M.J.; REECE, W.O. (Eds.) Dukes fisiologia dos animais domésticos. Rio de Janeiro: Guanabara, 1996. p.572-614.

DUKES, H.H.; SWENSON, M.J. Fisiologia de los animales domesticos. 4.ed. Madrid: Aguillar, 1978. 1864p.
ENCARNAÇ̃̃O, R.O. Estresse e produção animal. In: CICLO INTERNACIONAL DE PALESTRAS SOBRE BIOCLIMATOLOGIA ANIMAL, 1., 1989, Jaboticabal. Anais... Jaboticabal: FUNEP, 1989. p.111-29.

FRASER, H.M., McNEILLY, A.S. Effect of chronic inhibition of thyrotrophin releasing hormone on the thyroid hormones and prolactin in the ewe. Journal Endocrinology, v.87, n.2, p.37-38, 1980.

GENUTH, S.M. Sistema endócrino. In: BERNE, R.M.; LEVY, M.N. (Eds.) Princípios de fisiologia. Rio de Janeiro: Guanabara, 1991. p.476-497.

HAHN, G.L. Shelter engineering for cattle and other domestic animals. In:___ Progress in animal biometeorology. Amsterdam: Swets \& Zeitlinger, 1976. v.1. p.175-186.

HARGREAVES, A.L.,; HUTSON, G.D. The stress response in sheep during routine handling procedures. Applied Animal Behaviour Science, v.26, n.1, p.83-90, 1990.

INGRAHAM, R.H.; STANLEY, R.W.; WAGNER, W.C., Seasonal effects of tropical climate on shaded and non shaded cows as measured by retal temperature, adreanl cortex hormone, thyreoid hormone and milk production. American Journal Veterinary Research, v.40, p.1792-1797, 1979.

JOHNSON, D.E. Principal components analysis. In:_Applied multivariate methods of data analyses. London: Duxbury Press, 1998. p.93-146.

KOLB, E. Fisiologia Veterinaria. 2.ed. Zaragoza: Acribia, 1976. $1115 \mathrm{p}$.

MAREK, J.; MOCSY, J. Tratado de diagnóstico clínico de las enfermedades internas de los animales domésticos. 2.ed. Barcelona: Labor, 1963. 147p.

MANLY, B.F.J. Principal components analysis. In: Multivariate statistical methods. 2.ed. London: Chapman \& Hall, 1994. p.76-92.

McDONALD, L.E. Veterinary endocrinology and reproduction 3.ed. Philadelphia: Lea \& Febiger, 1980. p.173-174.

McFARLANE, A.; COGHLAN, J.; TRESHAM, J. et al. Corticotropin-releasing factor alone, but not arginine vasopressin alone, stimulates the release of adrenocorticotropin in the conscious intact sheep. Endocrinology, v.136, n.5, p.1821-1827, 1995.

McNABB, A.F.M. Thyroid hormones, their activation, degradation and effects on metabolism. In: CONFERENCE METABOLIC MODIFIERS, 1995, Amsterdam. Proceedings... Amsterdam: Elsevier, 1995. p.1773S-1776S. MENDES, M.A.; LEÃO, M.I.; SILVA, J.F.C. et al. Efeito da temperatura ambiente e do nível de energia da ração sobre os consumos de alimento e água e algumas variáveis fisiológicas de ovinos. Revista da Sociedade Brasileira de Zootecnia, v.5, n.2, p.173-187, 1976.

MINTON, J.E.; APPLE, J.K.; PARSONS, K.M. et al. Stressassociated concentrations of plasma cortisol cannot account for reduced lymphocyte function and changes in serum enzymes in lambs exposed to restraint and isolation stress. Journal Animal Science, v.73, n.2, p.812-817, 1995.

NASCIMENTO, M.R.B.M. Efeito da variação da temperatura ambiente sobre os níveis séricos de $3,5,3$, triiodotironina $\left(T_{3}\right)$ e tiroxina $\left(T_{4}\right)$ em ovinos corriedale. Jaboticabal: Universidade Estadual Paulista, 1994. 44p. Dissertação (Mestrado em Zootecnia) - Universidade Estadual Paulista, 1994.

NATIONAL RESEARCH COUNCIL - NRC. Nutrient requirements of sheep. 6.ed. Washington, D.C.: National Academy of Science, 1985. 99p. 
NAZKI, A.R.; RATTAN, P.J.S. Some hormonal and biochemical characteristics of blood in sheep as related to different seasonal enviroments. Indian Veterinary, v.68, p.28-32, 1991.

ROMAN-PONCE, H.H.; THATCHER, W.W.; WILCOX, C.J. Hormonal interrelationships and physiologivcal response of lactation dairy cows to a shade manegement system in a subtropical environment. Theriogenology, v.16, p.139-154, 1981 .

RE, R.N.; KOURIDES, I.A.; RIDGEMAY, E.C. et al. The effect of glucocorticoid administration on human pituitary secretion of thyrotropin and prolactin. Journal Clinical Endocrinology Metabolism, v.43, p.338-346, 1976.

REAP, M.B.S.; CASS, C.M.S.; HIGHTOWER, D.D.V.M. Thyronine and triiodothyronine levels in ten species of animals. Southwest Veterinarian, v.31, n.1, p.31-34, 1978.

ROSS, T.T.; GOODE, L.; LINNERUD, A.C. Effects of high ambient temperature on respiration rate, rectal temperature, fetal development and thyroid gland activity in tropical and temperate breeds of sheep. Theriogenology, v.24, n.2, p.259-269, 1985.

RUCKEBUSCH, Y.; PHANEUF, L.P.; DUNLOP, R. Physiology of small and large animals. Philadelphia: Decker, 1991. p.513-520.

SALEM, M.H.; YOUSEF, M.K.; EL-SHERBINY, A.A. et al. Physiology of sheep and goat in the tropics. In: YOUSEF,M.K. Animal production in the tropics. New York: Praeger, 1982. p.32-45.

SALEM, M.H.; EL-SHERBINY, A.A.; KHALIL, M.H. et al. Diurnal and seasonal rhythm in plasma cortisol, trodothyronine and thyronine as effected by the wool coat in Barki sheep. Indian Journal Animal Science, v.61, n.9, p.946-951, 1991.

SANO, H.; TAKAHASHI, K.; AMBO, K. et al. Turnover and oxidation rates of blood glucose and heat production in sheep exposed to heat. Journal Dairy Science, v.66, p.856-861, 1983.

STATISTICAL ANALYSES SYSTEM - SAS. SAS user's guide: statistics. Cary: 2001. 956p.
SILVA, R.G. Introdução à bioclimatologia animal. São Paulo: Nobel, 2000. 286p.

SILVA, R.G.; GAUDIOSI, M.C. Termólise evaporativa em ovinos sob altas temperaturas. In: CONGRESSO BRASILEIRO DE BIOMETEOROLOGIA, 1.,1995, Jaboticabal. Anais.. Jaboticabal, 1995. p.193-203.

STERLING, K.; LAZARUS, J.H. The thyroid and its control. Annual Review Physiology, v.39, p.349-371, 1977.

SUTHERLAND, R.L.; IRVINE, C.H.G. Total plasma thyroxine concentrations in horses, pigs, cattle, and sheep: anion exchange resin chromatography and ceric-arsenite colorimetry. American Journal Veterinary Research, v.34, n.10, p.1261-1265, 1973.

URIBE-VELASQUEZ, L.F.; OBA, E.; BRASIL, L.H.A. et al. Concentrações plasmáticas de cortisol, hormônios tireoideanos, metabólitos lipidicos e temperatura corporal de cabras alpinas submetidas ao estresse térmico. Revista Brasileira de Zootecnia, v.6, p.1123-1130, 1998.

WALLACE, A.L.C. Variations in plasma thyroxine concentrations throughout one year in penned sheep on a uniform feed intake. Australian Journal Biology Science, v.32, p.371-374, 1979.

WEBSTER, J.R.; MOENTER, S.M.; WOODFILL, C.J.I. et al. Role of the thyroid gland in seasonal reproduction. II. Thyroxine allows a season specific suppression of gonadotropin secrection in sheep. Endocrinology, v.129, n.1, p.176-183, 1991.

YOUSEF, M.K.; KIBLER, H.H.; JOHNSON, H.D. Thyroid activity and heat production in cattle following sudden ambient temperature changes. Journal of Animal Science, v. 26, n.1, p.142-148, 1967.

Recebido em: 05/09/03

Aceito em: 15/05/05 